\title{
Flocculation Behavior of Mature Fine Tailings: Decreasing Water Use in Oil Sands Extraction
}

\author{
V. A. Munoz*, G. R. D. Elliott*, A. Demoz*, and R. J. Mikula* \\ *CanmetENERGY, Devon Research Centre, Natural Resources Canada, 1 Oil Patch Drive Devon, \\ Alberta, Canada, T9G 1A8
}

A by-product of the oil sands extraction process in Northern Alberta is the accumulation of large volumes of mature fine tailings (MFT). Mature fine tails are the clay and silt component of the bitumen extraction process and are typically 35 to $40 \%$ solids by weight. This material collects in the bottom of large recycle water ponds as the extraction tailings slurry settles to form a water layer for recycle back to the extraction process. Because of its relatively low solids content, a significant portion of the make up water from the Athabasca River is due to water accumulating with this fluid or mature fine tailings component. In order to increase the proportion of water recycled and reused, and therefore decrease water withdrawal from the Athabasca River, a variety of tailings dewatering options are being tested and commercialized. Mature fine tails consist of roughly $20-30 \%$ solids, 1 $3 \%$ bitumen and $70-80 \%$ water creating a fluid material that is difficult to treat. In order to remove the water, the relatively stable (to consolidation) clay structure must be disturbed or modified. Although inorganic coagulants (gypsum, carbon dioxide, alum) can be used, these all affect the resulting recycle water chemistry, sometimes to the detriment of the extraction process. As a result, organic polymers are being investigated in order to control the flow behaviour (rheology) of the clay and silt suspension, recover the water, and not affect the overall recycle water chemistry. Polymers can be used to flocculate the MFT to allow for faster dewatering and compaction to occur; however, unlike inorganic additives, it is crucial that the mixing conditions are optimal to prevent overshearing of the polymer, destruction of the flocc structure, and reduction in the ability to consolidate the solids and remove water.

Current tailings treatment technologies include centrifugation, rim ditching, and thin lift dewatering. All of them require a chemical modification of the clay structure in order to optimize dewatering rates. Thin lift consists of creating a MFT-polymer solution that is deposited in thin layers on a horizontal surface. Once a layer has dried another layer is deposited on top of the last and this process is repeated to build up a deposit. Under ideal conditions, a significant amount of the water is recovered as the deposit is pumped along the beach. In contrast, rim ditching consists of a MFTpolymer solution that is deposited in a pit or containment area. The pressure of the material above helps to squeeze water out of the deposit. When enough strength is created in the clay suspension, a continuous ditch is created around the edge of the deposit to allow for accumulation of the water pushed from the pore spaces, which is collected and removed. Centrifugation of the polymer treated fluid fine tailings uses the high g-forces of a centrifuge to separate the water from the clays. For all three of these MFT treatment technologies a critical balance between the amount of polymer and the mixing conditions dictate the effectiveness of this ability to release water for drying. When mixing conditions are ideal, water release is maximized. This can be measured directly by quantifying the volume of water collected on the top of the test sample, or indirectly by measuring how quickly the water will drain from the sample in a capillary suction test. If the flocculant and clay system is under-mixed or over-mixed mix, dewatering is not optimum (see FIG. 1). The flocculated clay structure that corresponds to the optimum in dewatering rate can be observed directly using 
microscopic techniques, as shown in FIG. 2. By understanding the nature of the optimum flocculated structure, it will be possible to more quickly optimize the shear or mixing regime that maximizes dewatering rates.

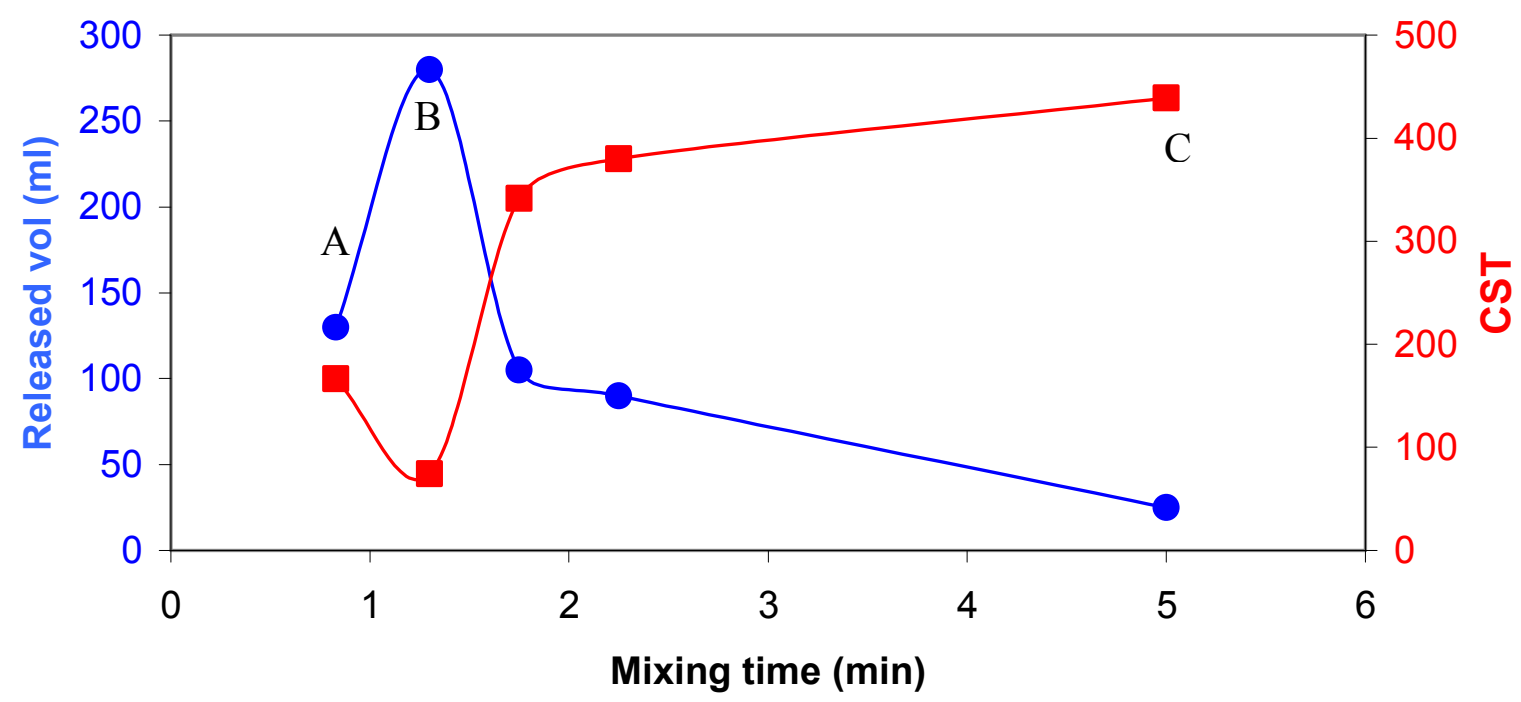

FIG. 1. The relationship between mixing time and dewatering rate. The dewatering rate is determined by the volume of water released and by how rapidly water drains from the sample (CST). Letters indicate the times when samples were taken to obtain the images in FIG. 2.

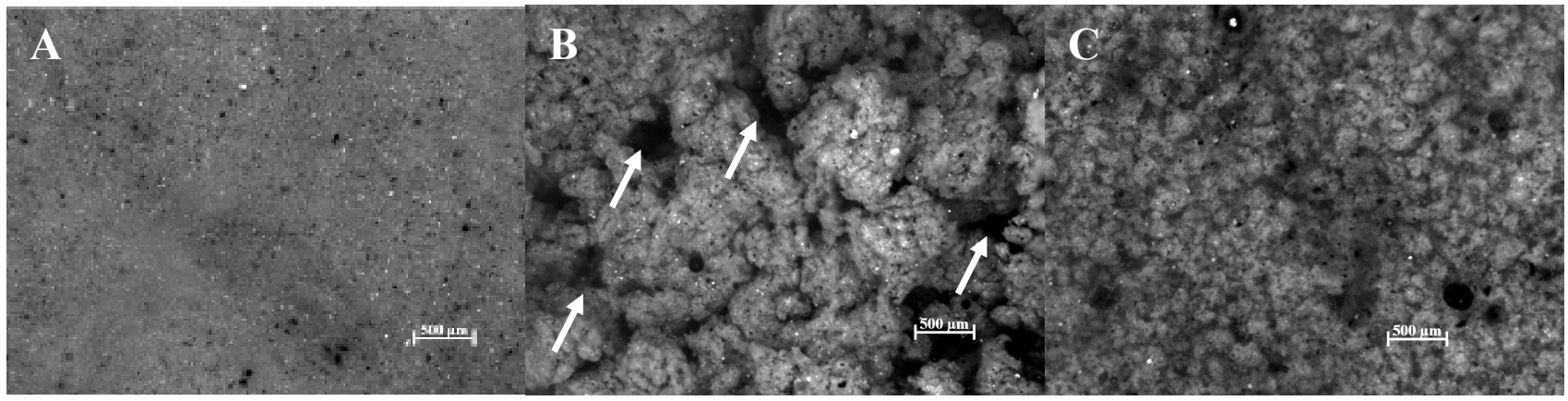

FIG 2. Flocculation behavior of the (A) mature fine tails, (B) optimized mixing conditions with polymer for maximal dewatering, and $(\mathrm{C})$ over-shearing of the polymer. The optimal mixing conditions produced large floccs and the formation of water channels which help to dewater the mixture. Arrows indicate free water channels forming between clumps of flocculated mature fine tails with polymer. When the mature fine tails-polymer mixture was over mixed there is a breakdown in the flocc structure and a reduction or elimination of the water channels. 